\title{
Experimental characterization of NBTI effect on pMOSFET and CMOS inverter
}

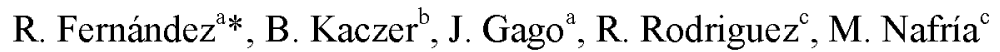 \\ ${ }^{a}$ Dept. d'Enginyeria Electrònica, Universitat Politècnica de Catalunya, Colom 1 E-08222-Terrassa \\ ${ }^{b}$ IMEC, Kapeldreef 75, B-3001 Leuven, Belgium \\ ${ }^{c}$ Dept. d'Enginyeria Electrònica. Universitat Autònoma de Barcelona, Edifici Q, E-08193 Bellaterra \\ "Corresponding author: +34 9373980 89; Fax: +34 9373980 16; e-mail: rfernan@eel.upc.edu
}

\begin{abstract}
In this paper, an experimental characterization of Negative Bias Temperature Instability (NBTI) effects on a single pMOSFET and CMOS inverter is done. The characterization has been performed for static and dynamic stresses with frequencies ranging from DC to GHz. The results show that NBTI produces a threshold voltage shift $\left(\Delta V_{T}\right)$ on pMOSFETs, which is frequency independent. For DC stress, this $\Delta V_{T}$ is double than in the $\mathrm{AC}$ case. In a CMOS inverter, NBTI produces a voltage transfer curve shift which is expected theoretically. As $\Delta V_{T}$, in a single pMOSFET, the voltage transfer curve shift in a CMOS inverter is frequency independent and almost double for DC stress in comparison to the $\mathrm{AC}$ case.
\end{abstract}

\section{INTRODUCTION}

The Negative Bias Temperature Instability (NBTI) is one of the main present and future reliability problems [1]. Nowadays, CMOS circuits are working from DC up to the $\mathrm{GHz}$ range, but not many dynamic NBTI (AC-NBTI) works have been reported [2]-[4] and are always focused on frequencies below a few MHz. In this paper, the NBTI effects on pMOSFET and CMOS inverter are experimentally investigated from $\mathrm{DC}$ to $\mathrm{GHz}$. In order to do that, on-chip circuits have been fabricated [5].

\section{EXPERIMENTAL}

The samples under test are pMOSFETs with an aspect ratio of $2 \mu \mathrm{m} / 0.13 \mu \mathrm{m}$ and CMOS inverters with aspect ratios of $3 \mu \mathrm{m} / 0.13 \mu \mathrm{m}$ and $6 \mu \mathrm{m} / 0.13 \mu \mathrm{m}$, for the nMOSFET and pMOSFET respectively. Fig. 1 depicts the stress setup. A constant voltage of $0 \mathrm{~V}$ or a square signal from $0-2 \mathrm{~V}$ were applied to the gate/inverter input for the static (DC) and dynamic (AC) stresses, respectively. In both cases, $2 \mathrm{~V}$ were applied to the rest of terminals, Depending on the stress frequency, an external pulse generator (DC-MHz) or on-chip stress circuits (MHz-GHz) have been used to provide the $\mathrm{AC}$ stress waveform. All the measurement have been done at $125^{\circ} \mathrm{C}$ at exponentially increasing periods of time.

The stress-measurement sequence is shown in Fig.2. First of all, the $I_{D}-V_{G}$ pMOSFET characteristic is measured (the Voltage Transfer Curve, VTC, in case of CMOS inverter), the data are saved and the stress is applied. After stress, the $I_{D}-V_{G}$ transistor curve (or the VTC of the inverters) are again measured and the data saved. If the sequence is not finished the stress time is exponentially increased and the stress is again applied. If the stress-measurement is finished the data are analysed and the threshold voltage shift $\left(\Delta \mathrm{V}_{\mathrm{T}}\right)$ is obtained.

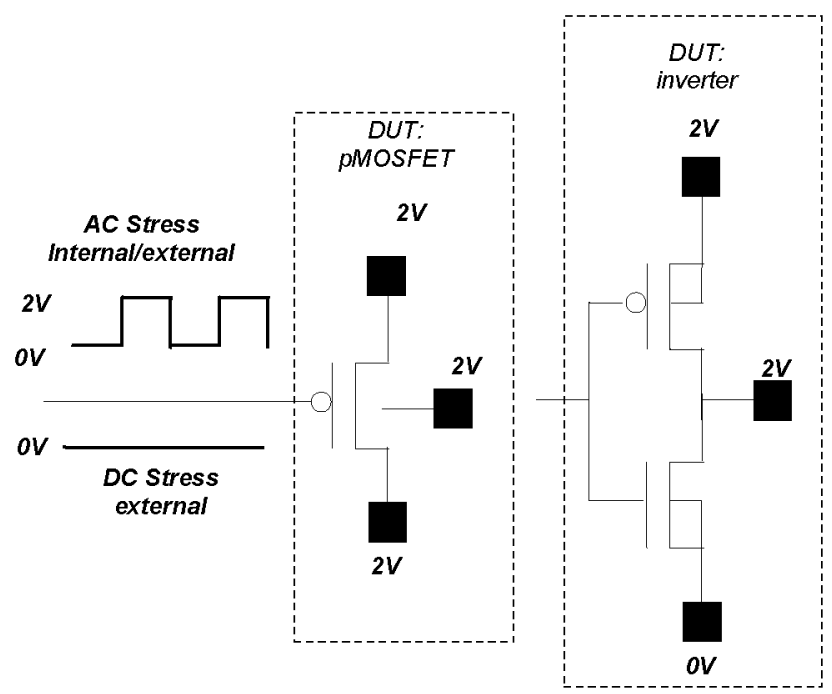

Fig. 1: Stress setup for static and dynamic NBTI measurement. Depending on the AC stress frequency, the stress signal is externally or on-wafer generated.

\section{RESULTS}

Fig. 3 shows the $I_{D}-V_{G}$ curve of a fresh and stressed pMOSFET. From these characteristics, $\Delta V_{\mathrm{T}}$ has been obtained for a constant current of $10 \mu \mathrm{A}$. For the stressed transistors the $I_{D}-V_{G}$ curve is shifted and therefore, $\left|V_{T}\right|$ increases,

Fig. 4 represents $\Delta \mathrm{V}_{\mathrm{T}}$ as a function of the stress time of pMOSFETs subjected to DC and AC (7.4MHz) NBTI stresses. In both cases, $\Delta V_{T}$ follows a power law dependence with the stress time and an exponent around 0.20 is obtained. However, in the case of AC stress, $\Delta \mathrm{V}_{\mathrm{T}}$ is almost half of the one obtained for the DC case. For instance, for $1000 \mathrm{~s}$ stress $\left(125^{\circ} \mathrm{C}\right.$ and $\left.2 \mathrm{~V}\right)$ the $\Delta \mathrm{V}_{\mathrm{T}}$ is around $40 \mathrm{mV}$ and $20 \mathrm{mV}$ for the $\mathrm{DC}$ and $\mathrm{AC}$ stresses respectively. . 
Proceedings of the 2009 Spanish Conference on Electron Devices - Feb 11-13, 2009. Santiago de Compostela, Spain.

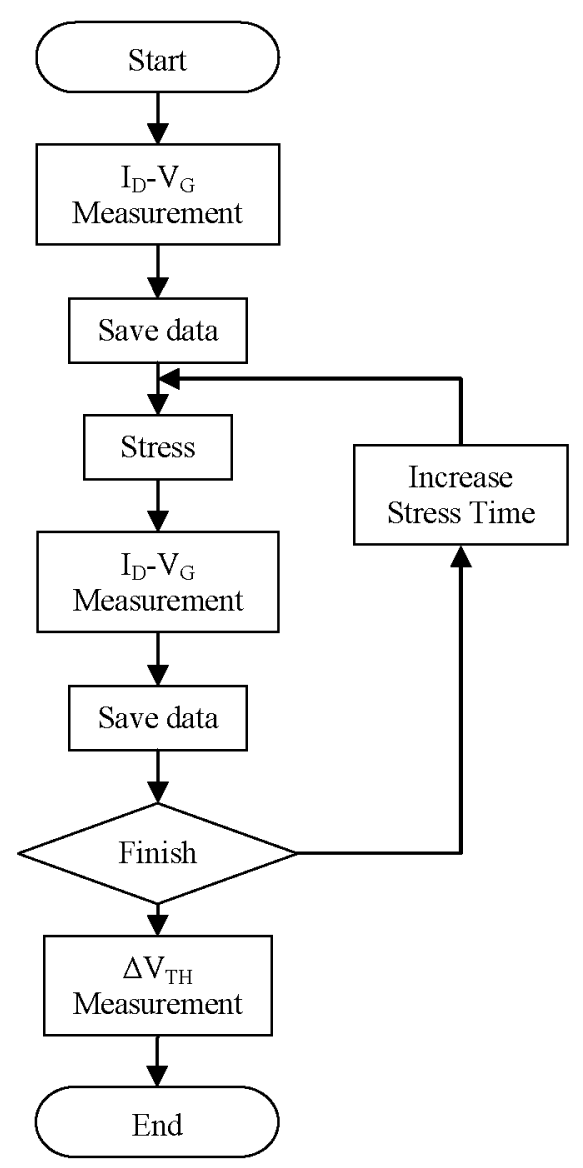

Fig. 2: Stress-measurement sequence.

In order to evaluate the $\Delta V_{T}$ dependence with the stress frequency, an AC stress swept has been applied to the gate. For frequencies from $1 \mathrm{~Hz}$ to $1 \mathrm{MHz}$ an external pulse generator was used. The $\mathrm{AC}$ stress voltages from $1 \mathrm{MHz}-2 \mathrm{GHz}$ were obtained from a on-wafer pulse generator [5]. Fig. 5 shows the $\Delta \mathrm{V}_{\mathrm{T}}$ frequency dependence for $1000 \mathrm{~s}$ stress $\left(125^{\circ} \mathrm{C}, 2 \mathrm{~V}\right)$. The plot confirms that $\Delta V_{\mathrm{T}}$ is independent of frequency in the wide range of $1 \mathrm{~Hz}-2 \mathrm{GHz}$ and the $\Delta \mathrm{V}_{\mathrm{T}}$ value obtained under $\mathrm{AC}$ stress is almost the half of the DC case. However, a low $\Delta V_{T}$ measured at $1 \mathrm{MHz}$ (the highest frequency measured using an external pulse generator) is observed. This lower value of $\Delta V_{T}$ can be attributed to the loss of the stress signal integrity due to a setup parasitic effect, which, on the other hand, could explain the frequency dependency reported in other works and demonstrates the need of on-chip NBTI characterizations.

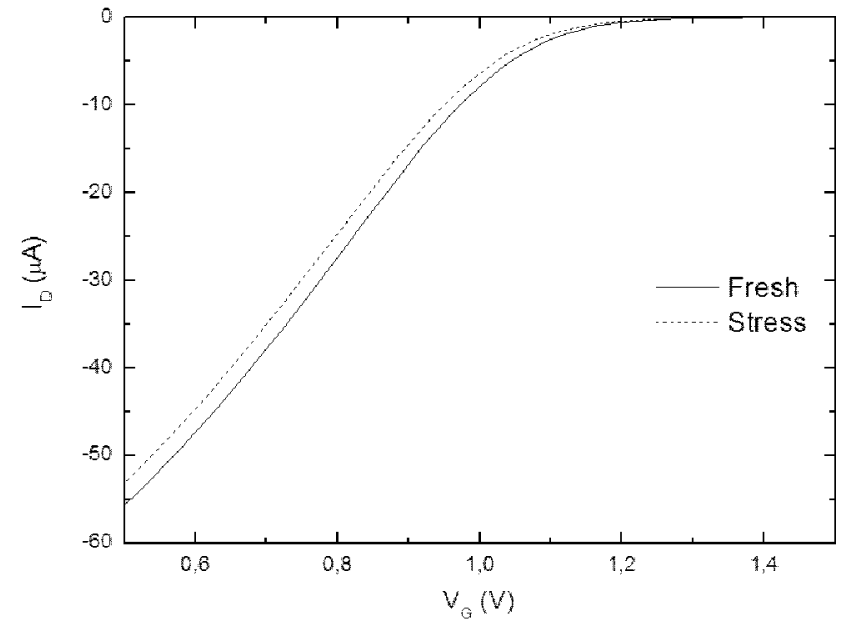

Fig. 3: $I_{D}-V_{G}$ characteristic of a fresh pMOSFET and after 1000 s NBTI stress $\left(125^{\circ} \mathrm{C}, 2 \mathrm{~V}\right)$.

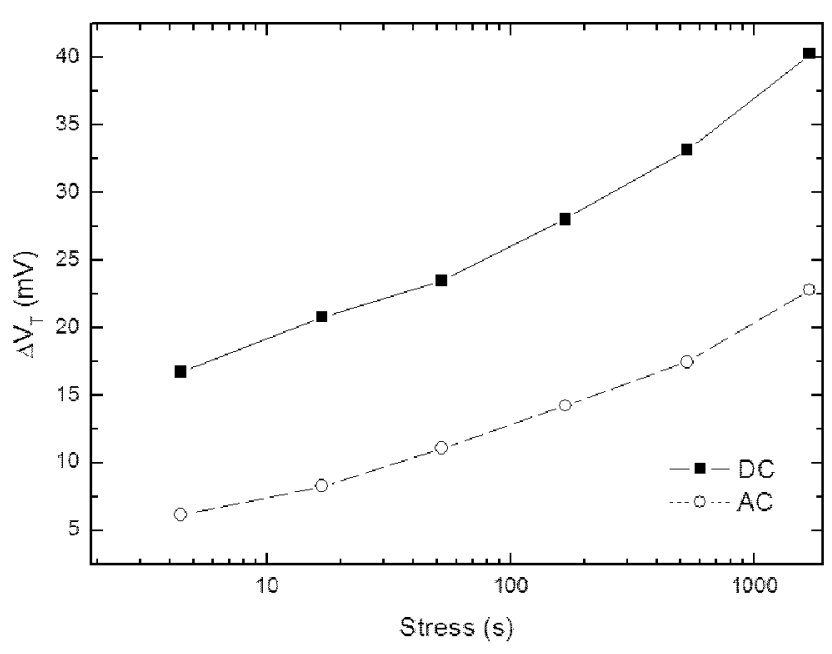

Fig. 4: $\Delta \mathrm{V}_{\mathrm{T}}$ as a function of $\mathrm{t}_{\text {stress }}$ for DC and AC NBTI stress conditions. In both cases $\Delta \mathrm{V}_{\mathrm{T}}$ follows a power law with exponents around 0.20 .

CMOS inverters have also been subjected to $\mathrm{DC}$ and $\mathrm{AC}$ stresses. In order to quantify the NBTI effects, the maximum gain point shift of the inverter voltage transfer curve has been used $\left(\Delta V_{\mathbb{N N}}\right)$. The theoretical relationship between $\Delta V_{\mathbb{N N}}$ and the inverter pMOSFET $\Delta \mathrm{V}_{\mathrm{TP}}$ is given by equation (1).

$$
\Delta V_{I N}=\frac{\Delta V_{T P}}{1+\sqrt{\frac{\mu_{N}(W / L)_{N}}{\mu_{P}(W / L)_{P}}}}
$$




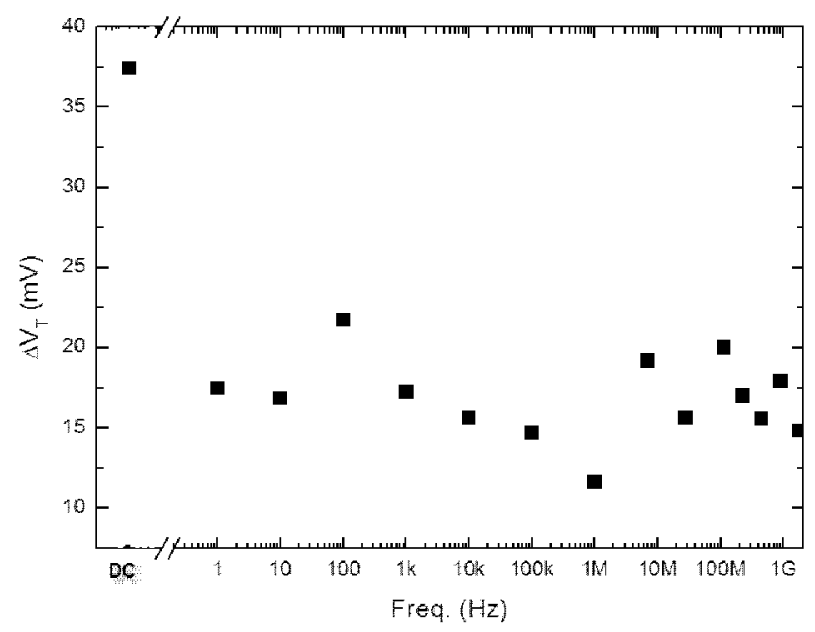

Fig. 5: Frequency dependence of $\mathrm{V}_{\mathrm{T}}$ shifts due to AC NBTI stress in a single pMOSFET

$\mu$ denote the carrier mobility and $\mathrm{W} / \mathrm{L}$ the transistor aspect ratio. The CMOS inverter under test has been designed in order to achieve the maximum gain point at $V_{D D} / 2$, so $\mu_{\mathrm{N}}(\mathrm{W} / \mathrm{L})_{\mathrm{N}}=\mu_{\mathrm{P}}(\mathrm{W} / \mathrm{L})_{\mathrm{P}}$. Forcing this condition, the ratio between $\Delta \mathrm{V}_{\mathrm{TP}}$ and $\Delta \mathrm{V}_{\mathbb{N}}$ is given by equation (2).

$$
\Delta V_{I N}=\frac{\Delta V_{T P}}{2}
$$

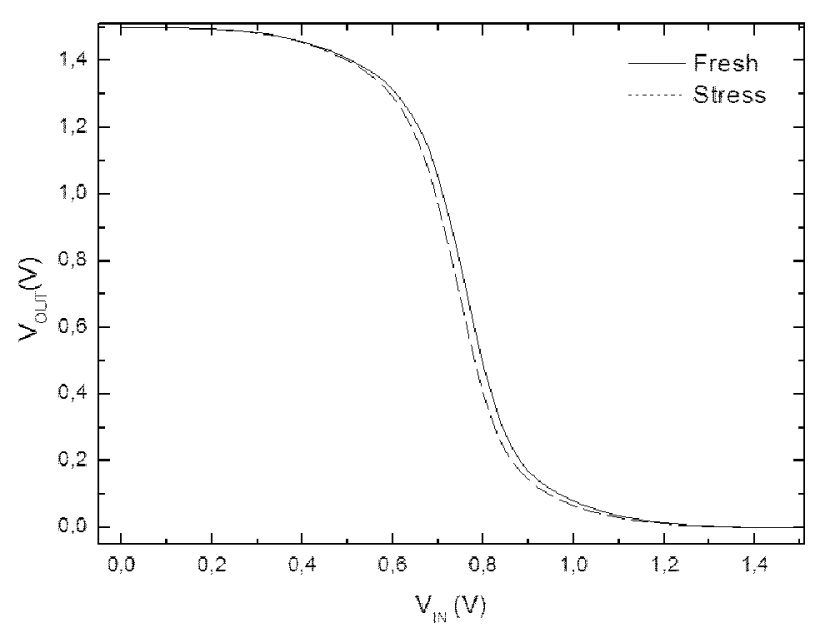

Fig. 6: The inverter voltage transfer curve before and after 1000 s NBTI stress $\left(125^{\circ} \mathrm{C}, 2 \mathrm{~V}\right)$.

In Fig. 6 the inverter voltage transfer curve before and after NBTI stress is plotted. A voltage transfer curve shift is produced after NBTI stress. Fig. 7 shows the $\Delta V_{\mathrm{IN}}$ frequency dependence. Again the shift produced due to $\mathrm{AC}$ stress is almost half of the DC case. Moreover, if $\Delta \mathrm{V}_{\mathrm{T}}$ of a pMOS transistor (Fig.5) is compared with $\Delta V_{\mathrm{IN}}$ of the inverter (Fig.7), it is observed that $\Delta \mathrm{V}_{\mathrm{IN}}$ is almost half of $\Delta \mathrm{V}_{\mathrm{T}}$, which is predicted theoretically by equation (2). This result confirms that the NBTI impact on CMOS inverter functionality is only due to the NBTI effect on the single inverter pMOSFET.

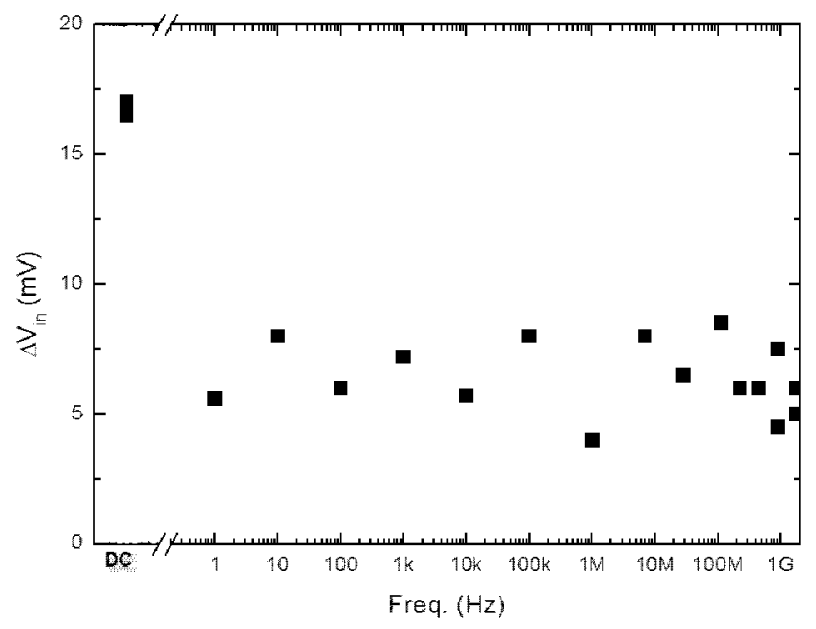

Fig. 7: Frequency dependence of the inverter maximum gain point shift, $\Delta \mathrm{V}_{\mathrm{IN}}$, due to AC NBTI stress.

\section{CONCLUSION}

Experimental characterization of NBTI effect on single pMOSFETs and CMOS inverters has been done. The results indicate that i) the NBTI impact on transistor performance subjected to AC stress is almost half of the DC case. ii) $\mathrm{AC}$ NBTI is independent of frequency in the entire $1 \mathrm{~Hz}-2 \mathrm{GHz}$ range iii) the effect of NBTI in the CMOS inverter functionality is only due to NBTI effects on the inverter pMOSFET.

\section{ACKNOWLEDGMENT}

This work has been partially supported by the Spanish Government (TEC2004-00798/MIC and TEC2007-61582), the European Commission (Marie Curie Actions, APROTHIN Project) and the Generalitat de Catalunya (2005SGR-00061).

\section{REFERENCES}

[1] Reddy, V.; Krishnan, A.T.; Marshall, A.; Rodriguez, J.; Natarajan, S.; Rost, T.; Krishnan, $\mathrm{S}$.; Impact of negative bias temperature instability on digital circuit reliability, Proc. IRPS, p. 248 (2002).

[2] Abadeer, W.; Ellis, W.; Behavior of NBTI under AC dynamic circuit conditions, Proc. IRPS, p. 17 (2003).

[3] Chakravarthi, S.; Krishnan, A.; Reddy, V.; Machala, C.F.; Krishnan, S.; A comprehensive framework for predictive modeling of negative bias temperature instability, Proc. IRPS, p. 273 (2004).

[4] Alam, M.A.; A critical examination of the mechanics of dynamic NBTI for PMOSFETs, IEDM Tech. Digest, p. 345 (2003).

[5] Fernandez, R.; Kaczer, B.; Nackaerts, A.; Demuynck, S.; Rodriguez, R.; Nafria, M.; Groeseneken, G.; AC NBTI studied in the $1 \mathrm{~Hz}$ y $2 \mathrm{GHz}$ range on dedicated on-chip CMOS circuits, IEDM Tech. Digest, p. 1 (2006). 